\title{
Hybrid MVDR-LMS beamforming for massive MIMO
}

\author{
Yasmine M. Tabra' ${ }^{1}$, Bayan Mahdi Sabbar ${ }^{2}$ \\ ${ }^{1}$ Department of Information \& Communication, Information Engineering College, Al-Nahrain University, Iraq \\ ${ }^{2}$ Department of Control Engineering, Information Engineering College, Al-Nahrain University, Iraq
}

\begin{tabular}{l}
\hline \hline Article Info \\
\hline Article history: \\
Received Jan 29, 2019 \\
Revised Apr 30, 2019 \\
Accepted May 21, 2019 \\
\hline Keywords: \\
Beamforming \\
Least-mean-square \\
LTE-5G \\
Massive MIMO \\
MVDR \\
\hline
\end{tabular}

\begin{abstract}
With the high speed of communication in 5G, fast beamforming techniques need to be adopted. The training time required to form and steer the main lobes toward $5 \mathrm{G}$ multiple users must be short. Least-Mean-Square (LMS) training time is not suitable to work with in $5 \mathrm{G}$, but it has a good performance in forming multiple beams to large number of users and producing nulls in the interference direction. In this paper, A hybrid of MVDR BF and LMS BF was made to reduce the amount of time required to draw maximized power beams in exact direction. The proposed MVDR-LMS method can form thinner beams with $3 \mathrm{~ms}$ less than LMS. The power of beams is reduced by $10.2 \%$ from power level of MVDR, with side lobs power below $100 \mathrm{db}$. The performance of the proposed hybrid MVDR-LMS algorithm tested using MATLAB 2016a.
\end{abstract}

Copyright (C) 2019 Institute of Advanced Engineering and Science. All rights reserved.

\section{Corresponding Author:}

Yasmine M. Tabra,

Department of Information \& Communication,

Information Engineering College,

Al-Nahrain University, Iraq.

Email: yasminetabra@yahoo.com

\section{INTRODUCTION}

Massive Multiple-input Multiple-output (MIMO) is considered as a key technology in future 5G systems to improve the spectrum efficiency. $5 \mathrm{G}$ made use of large number of adaptive (smart antennas) at the base station (BS) [1]. The basic idea of beamforming is to focus energy towards a receiver. Traditionally access points were equipped with omni-directional antennas, named so because they send energy in all directions. Since they radiate waves in every direction, receiver antennas need not track every client but it comes with its own disadvantages. Alternatively, energy can be focused toward the particular receiver which is the principle of beamforming [2].

Beam forming is the process of combining the weighted of received signals on an array of sensors to improve the directionality. Adaptive beamforming is the ability of the beamformer to receive the signal only from the desired direction and to reject all other signals from undesired directions. The weight vector for the adaptive beamformer continuously changes based on some adaptive algorithm $[3,4]$.

The beamforming technique is used in smart antennas for transmitting and receiving signals in massive MIMO systems. Beamforming tries to produce patterns from the antenna's beam directed towards the target mobile terminal, while neglecting interference signals [5, 6]. Adaptive beamforming is more suitable for massive MIMO systems than fixed beamforming because of its ability to eliminate interference and reduce power consumption [5].

There are two types of adaptive algorithm are Blind and Non blind algorithm. Non blind adaptive algorithms require the statistical knowledge of the transmitted signal in order to converge to a weight solution, such as LCMV (Linearly Constrained Minimum Variance), and MVDR (Minimum Variance Distortionless Response) [7]. LCMV uses power constraints to selects optimum weight vectors to reduce the filter's response. LCMV are inappropriate for application to massive MIMO systems because of its low convergence rate. 
MVDR Beamforming algorithm selects the antenna elements weight while keeping a constant gain level of beam steered toward the desired direction. The properties of LCMV and MVDR indicate that MVDR is more suitable for massive MIMO systems than LCMV. MVDR is more convenient algorithms for massive MIMO systems [8].

The Non blind based adaptive algorithm are based on minimization of mean square error between received signal and reference signal, such as LMS (Least mean square) for smart antenna system. LMS uses a gradient based method of steepest decent and requiring reference signal and no knowledge channel [7, 9]. Beamforming could be classified into analogue beamforming, digital beamforming, and hybrid analogue/digital beamforming. Hybrid analogue/digital beamforming has been developed for massive MIMO systems to obtain the advantages of analogue and digital beamforming [10].

$5 \mathrm{G}$ requires a high frequency band signals for achieving an extremely high data rate. To make it possible mm-wave beamforming which is considered a wideband beamforming can be implemented [11]. Hybrid beamforming, which has emerged a low complexity and cost effective solution for millimeter wave communications, are the most promising approach in massive MIMO systems [11].

The optimal algorithms that satisfy the requirements of next-generation wireless communication systems can be one of the adaptive beamforming, such as MVDR, or a combination of two algorithms, to estimate accurately the DOA in terms of the azimuth and elevation angles (2D-DOA) [5]. Many researchers proposed beamforming methods that work with massive MIMO.

ALI et al. [5] classified the optimal beamforming techniques that are used in wireless communication and suggested an optimal beamforming technique for massive MIMO systems that satisfy the next-generation requirements.

Andreas F. et al. [10] made a survey of the various beamforming methods proposed in the literature and indicates that hybrid beamforming are the most promising approach for reducing the hardware cost and training overhead in massive MIMO systems.

S. Shahab et al. [13] evaluated the performance of MVDR by mean of beam pattern of two incoming signals and Signal to Interference plus Noise Ratio (SINR). The results showed that MVDR performance improves with the increasing number of elements. Also, the beam pattern depends on two factors: the number of elements and the spacing between array elements. The best inter element spacing obtained is $0.5 \lambda$ to avoid grating lobes and mutual coupling effects.

Noh et al. [14] demonstrated the use of hybrid beamforming, which are a mixture of digital and analogue beamforming, in massive MIMO systems.

In this paper a new hybrid method solution for massive MIMO was proposed. This hybrid method combines MVDR algorithm for its well-known null steering with LMS algorithm that don't require adjusting the weights of the array.

In this paper a beamforming algorithm was proposed. This method combines the adaptive blind beamforming algorithm, MVDR, with the non-blind beamforming LMS. The next two sections contain description of these two algorithms.

\section{RESEARCH MEHTODS}

\subsection{Minimum Variance Distortionless Response (MVDR) Beamforming}

The basic idea of the Minimum Variance Distortionless Response (MVDR) algorithm or Capon beamformer is to estimate the beamforming coefficients in an adaptive way by minimizing the output power of the beamformer under a single linear constraint on the response of the array towards the desired signal $[13,15]$. The MVDR beam former does not require the knowledge of the directions of the interferences for weight vector calculation. It requires only the direction of the desired signal. MVDR weight vector is given by: [16]

$$
w=\frac{R^{-1} a_{(\theta, \varphi)}}{a_{(\theta, \varphi)}^{H} R^{-1} a_{(\theta, \varphi)}}
$$

Where R array correlation matrix

$$
R=x(t) * x^{H}(t)
$$

and $a_{\theta}$ is the signal steer vector on array elements can be expressed as [13]

$$
a_{(\theta)}=\left[\exp \left(\frac{j 2(m-1) \pi d}{\lambda} \sin (\theta)\right)\right] \text { for } m=1,2, \ldots, M
$$


Thus, the beamformer weights are selected by maximum value, to select the element and minimum mean value of output power depending on the number of user inside the coverage while maintaining unity response in the look direction [17].

The output signal power of the array using optimum weight vector from MVDR beamforming method is given by MVDR spatial spectrum for angle of arrival estimated by detecting the peaks in this angular spectrum as $[18,19]$

$$
P_{M V D R}(\theta, \varphi)=\frac{1}{a^{H}(\theta, \varphi) R^{-1} a(\theta, \varphi)}
$$

The standard Capon beamformer has better resolution than the conventional beamformer provided that the array steering vector corresponding to the signal of interest is accurately known [20].

\subsection{LMS}

Least Mean Square (LMS) algorithm depends on continuous update to its parameters. LMS algorithm calculates the weight vector using repeated procedure. It makes use of the available data to estimate gradient vector. Weights should be in the negative direction of the gradient vector which produces minimum square error [21]. The following equations describes the LMS operation [22]

$$
\begin{aligned}
& y(n)=w^{H}(n) x(n) \\
& e(n)=d(n)-y(n) \\
& w(n+1)=w(n)+\Delta \cdot x(n) \cdot e(n)
\end{aligned}
$$

Where, $y(n)=$ array output, $x(n)=$ input data vector, $d(n)=$ reference signal, $w(n)=$ weight vector, $e(n)=$ error signal, and $\Delta=$ step size $\left(0<\Delta<\lambda_{\max }\right)$, where $\lambda_{\max }$ is the largest Eigenvalue of correlation matrix $\mathrm{R}$ as in (2)

Any change to the input data characteristics require changing the filter weights to adapt to these changes. The target of the filter is that $\mathrm{e}(\mathrm{n})$ becomes zero $[23,24]$.

\section{SYSTEM MODEL}

The Massive MIMO system model is represented uniform Rectangular array (URA) with M element in the $\mathrm{X}$-axis and $\mathrm{N}$ element in the $\mathrm{y}$-axis and spacing between elements $\mathrm{d}$. $\mathrm{T}$ different sources transmit signal $\mathrm{S}_{\mathrm{i}}$ at the same time, where $1 \leq \mathrm{i} \leq \mathrm{T}$. As in Figure 1 .

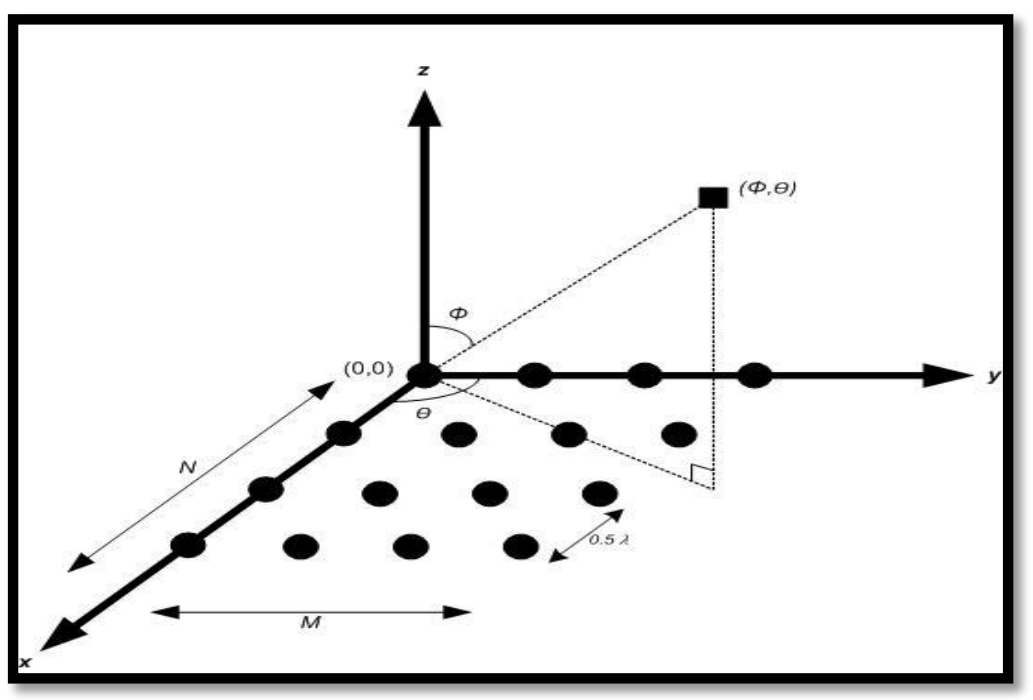

Figure 1. Uniform rectangular array (URA) 
The received signal at $(m, n)$ th antenna for $" 1 \leq m \leq M, 1 \leq n \leq N "$, is represented by the following:

$$
\mathrm{X}_{\mathrm{m}, \mathrm{n}}=\sum_{\mathrm{i}=1}^{\mathrm{T}} \mathrm{S}(\mathrm{i}) \mathrm{e}^{\mathrm{j}\left((\mathrm{m}-1)\left(\frac{2 \pi \mathrm{d}}{\lambda}\right) \sin \theta_{\mathrm{i}}+(\mathrm{n}-1)\left(\frac{2 \pi \mathrm{d}}{\lambda}\right) \cos \theta_{\mathrm{i}} \sin \varphi_{\mathrm{i}}\right)}+\mathrm{W}_{\mathrm{m}, \mathrm{n}}
$$

After collecting the received signal in $\mathrm{M}$ x N matrix, the final received signal from all sources could be represented by two equations using the Separated Steering Matrix method (SSM) in [25]

$$
\mathrm{X}_{\mathrm{u}}=\mathrm{A}_{\mathrm{u}} \mathrm{S}+\mathrm{W} \text { and } \mathrm{X}_{\mathrm{v}}=\mathrm{A}_{\mathrm{uv}} \mathrm{S}+\mathrm{W}
$$

Where, $\mathrm{S}=\left[\mathrm{S}_{1}, \mathrm{~S}_{2}, \ldots, \mathrm{S}_{\mathrm{T}}\right]^{\mathrm{T}}, \mathrm{W}$ is $\mathrm{M} \times \mathrm{N}$ noise matrix, $\mathrm{A}_{\mathrm{u}}$ represents the steering matrix with the elevation angle $\theta$, While the $\mathrm{A}_{\mathrm{uv}}$ contain both azimuth and elevation angles.

$$
A_{u}=\begin{array}{cccc}
1 & 1 & \cdots & 1 \\
\mathrm{e}^{\mathrm{j}\left(\mathrm{u}_{1}\right)} & \mathrm{e}^{\mathrm{j}\left(\mathrm{u}_{2}\right)} & \cdots & \mathrm{e}^{\mathrm{j}\left(\mathrm{u}_{\mathrm{T}}\right)} \\
\vdots & \vdots & \cdots & \vdots \\
\mathrm{e}^{\mathrm{j}\left((\mathrm{M}-1) \mathrm{u}_{1}\right.} & \mathrm{e}^{\mathrm{j}\left((M-1) \mathrm{u}_{2}\right.} & \cdots & \mathrm{e}^{\mathrm{j}\left((M-1) \mathrm{u}_{\mathrm{T}}\right.}
\end{array}
$$

and

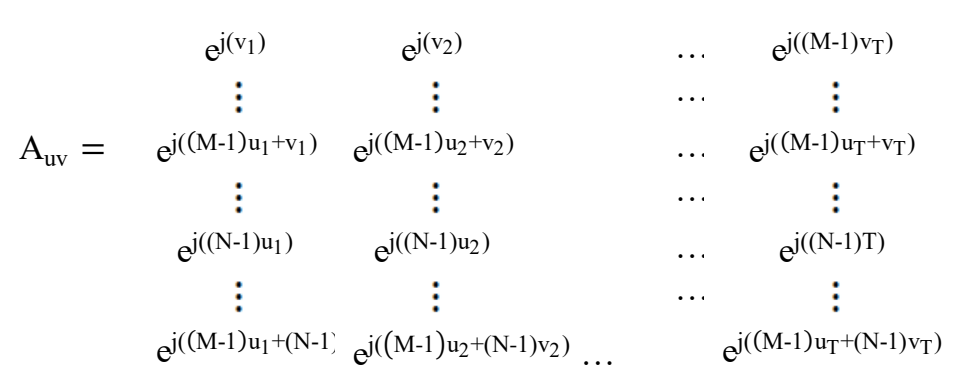

The array factor (AF) of M elements in X-dimension can be expressed as,

$$
A F(\theta)=\sum_{m=0}^{M-1} A_{u_{m}} w_{u_{m}}
$$

And (AF) of $\mathrm{N}$ elements in $\mathrm{y}$-dimension can be expressed as,

$$
A F(\varphi)=\sum_{n=0}^{N-1} A_{u v_{n}} w_{u v_{n}}
$$

Normalized Array factor is,

$$
A F_{\text {norm }}=\frac{A F}{A F_{\max }}
$$

\section{PROPOSED METHOD}

The proposed beamforming method combines two adaptive beamformers. This beamformer work in cascade form of two stages, where the first stage is the MVDR Beamformer and the second stage is LMS beamformer. These two beamformers takes feedback information from the proposed SSM DOA for continuous determining and acquisition of users connected to BS. Figure (2) shows a block diagram of beamformer. MVDR belongs to the blind beamformers that doesn't require knowledge of a reference signal and, therefore, there is no need to adjust the weights of the array. It has one limitation that it requires a prior knowledge about the direction of the interference signals. This causes a tangle in apply since the directions of interference signals vary over time.

To overcome this shortage, here comes the role of the second beamformer. The LMS beamformer is an adaptive beamformer belongs to the non-blind beamformers and it adaptively varies the antenna array pattern to produce nulls toward the direction of interference. 


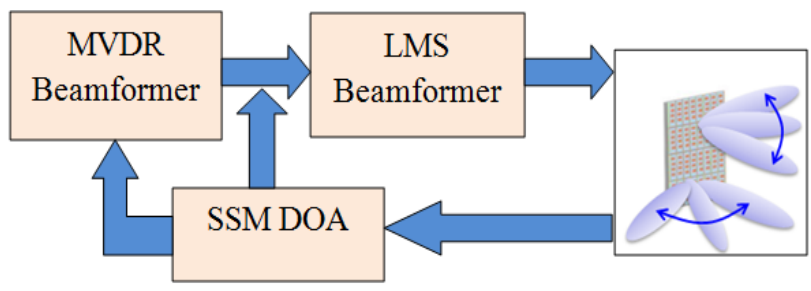

Figure 2. Block diagram of hybrid MVDR-LMS

Hybrid MVDR-LMS beamformer, will benefit from constant initial weights of that MVDR in equation (4) to provide fast start in catching the initial user location, while LMS will use equation (7) to provide the ability to keep track to user direction and produce null in the interference direction. i.e. this proposed algorithm will get use from the good specifications of both MVDR and LMS. The algorithm is described by mean of flowchart as shown in Figure 3.

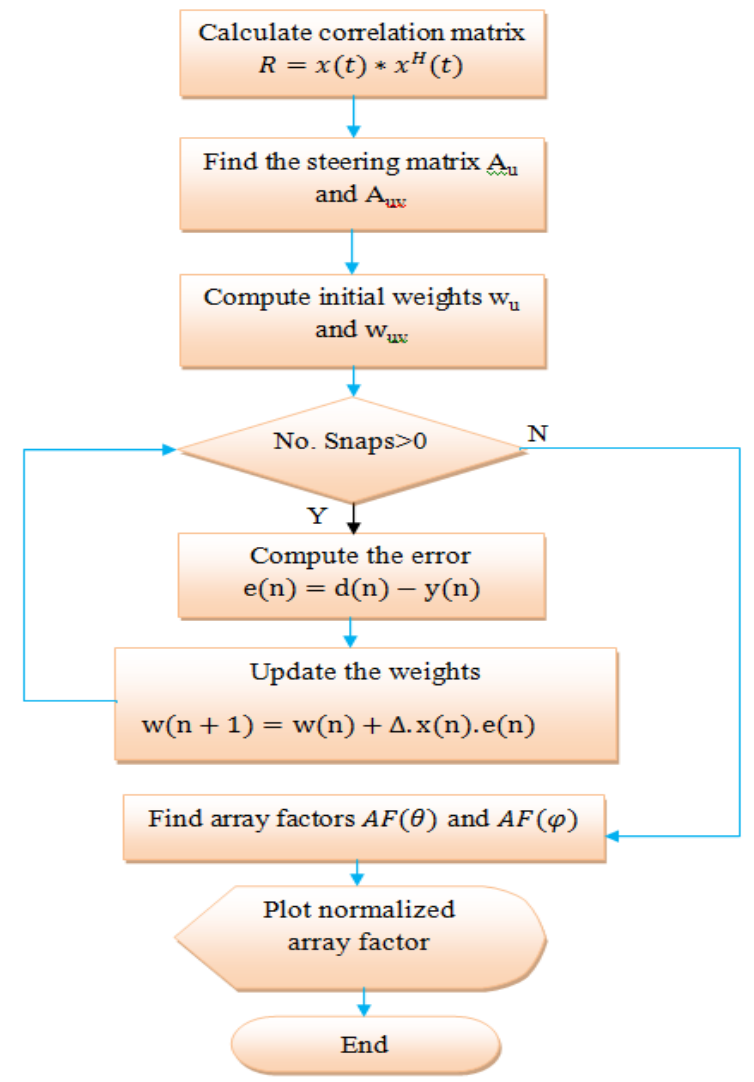

Figure 3. Flowchart of hybrid MVDR-LMS

\section{RESULTS AND DISCUSSION}

To show the performance of the proposed hybrid MVDR-LMS algorithm, MATLAB 2016a is used. A massive MIMO model with 128 antennas in the $\mathrm{x}$-dimension and $\mathrm{y}$-dimension was used with $0.5 \lambda$ spacing between elements, number of snapshots 50, and carrier frequency $26 \mathrm{GHz}$.

Test is carried with four signals received at the array elements with azimuth angle $=\{-10,10,20$, $30\}$ and elevation angle $=\{10,20,30,40\}$ with 50 snapshots. The direction of arrival was estimated using 2D-MUSIC using separated steering matrix (SSM) [25]. Figure (4) shows beamforming using MVDR, LMS, and hybrid MVDR-LMS for elevation and azimuth angles using 50 snapshots. 

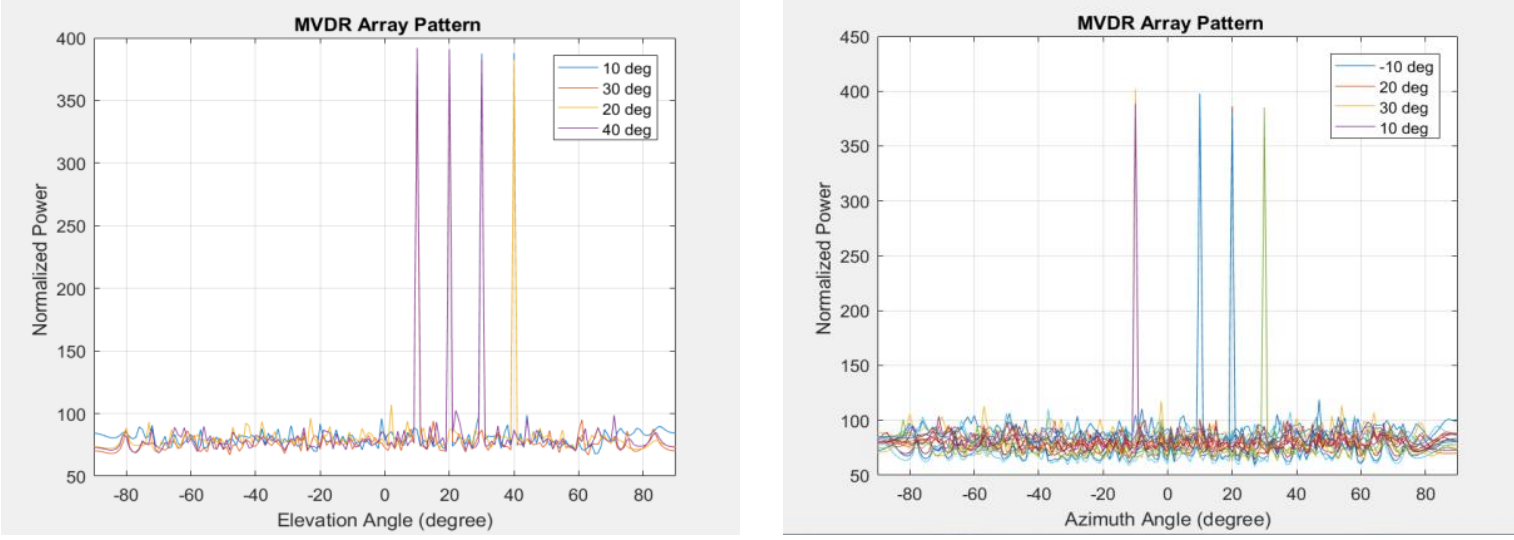

(a) MVDR beamformer
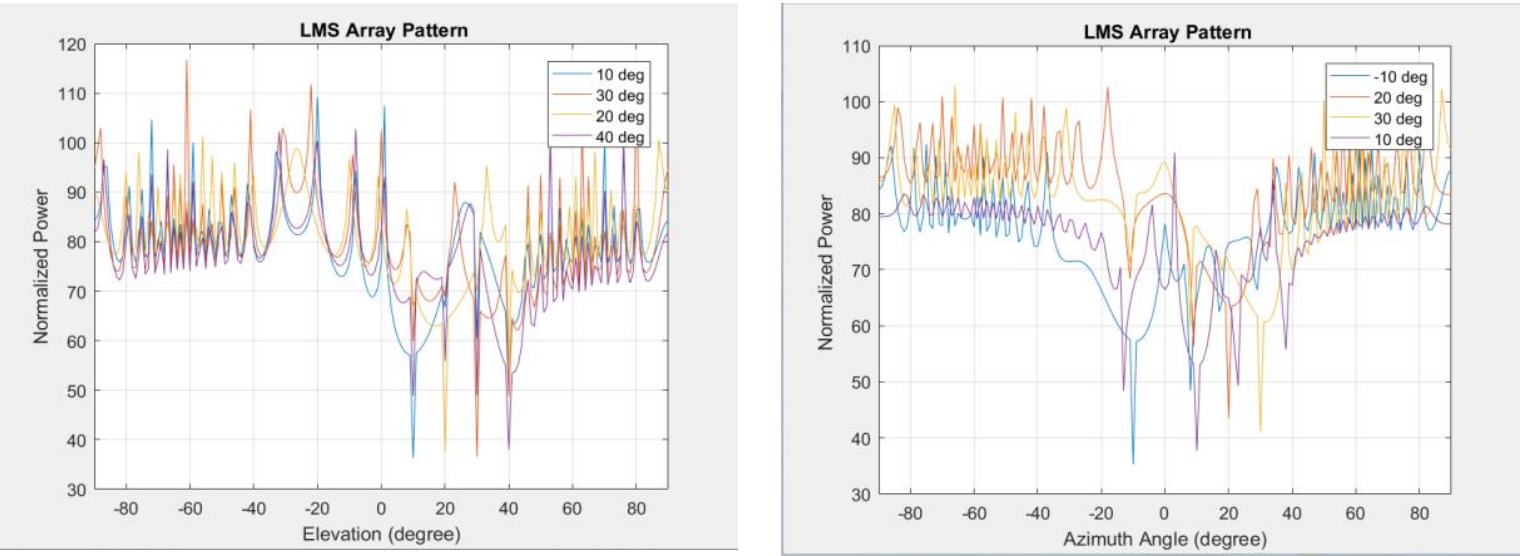

(b) LMS beamformer
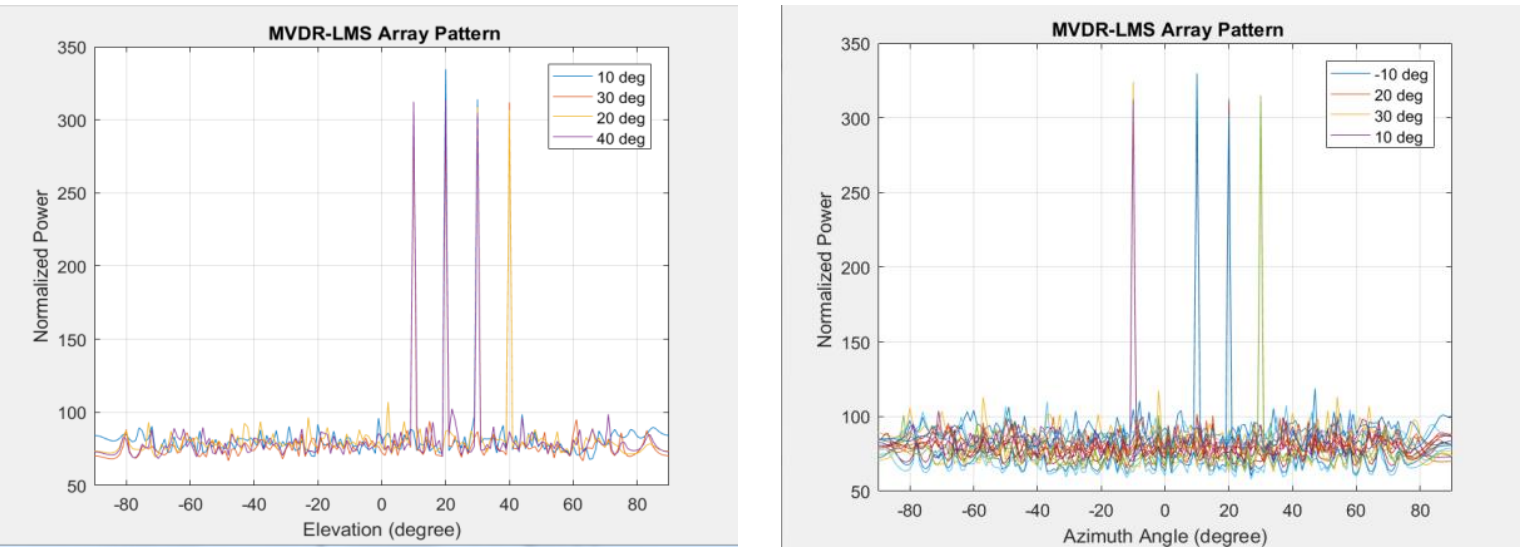

(c) MVDR-LMS beamformer

Figure 4. Different beamformers using 50 snapshots

The figure above shows that all beamformers are able to form peaks in the desired direction, but hybrid MVDR-LMS produced narrower beams with power higher than that of MVDR and LMS individually. Even when the number of snapshots is reduced to 10 snapshots, it can produce thinner beams with maximized power as shown in Figure 5. 


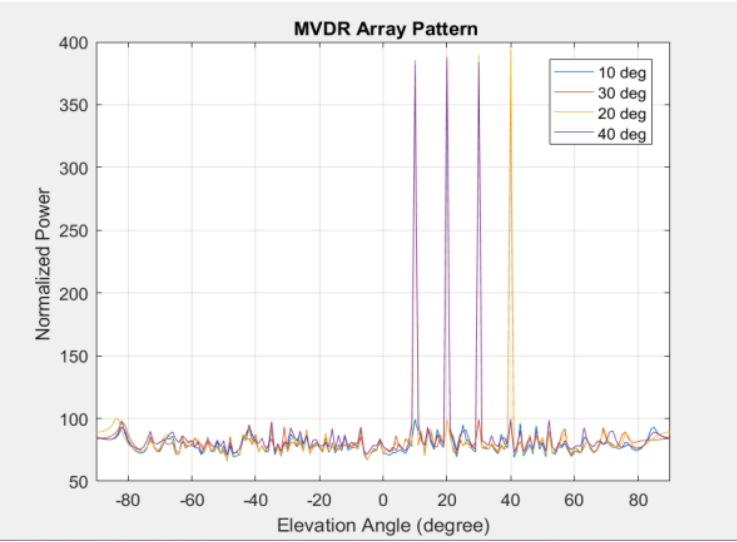

(a) MVDR

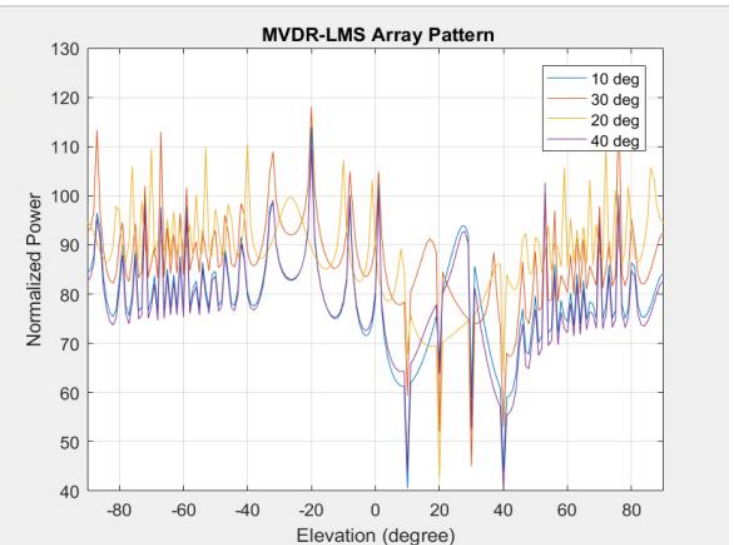

(b) LMS

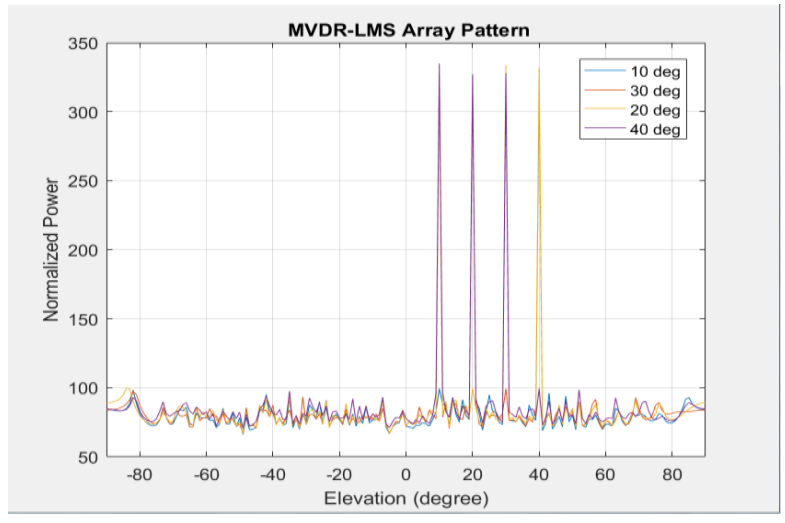

(c) MVDR-LMS

Figure 5. beamformers with no. snaps $=10$

A test for the simulation time required for beamforming in MVDR, LMS and MVDR-LMS with different number of snapshots was made. Table 1 shows the comparison in time between these algorithms.

Table 1. DOA and Beamforming Time

\begin{tabular}{ccc}
\hline Beamformer & Number of snapshots & Time in sec \\
\hline MVDR & & 0.811 \\
LMS & \multirow{2}{*}{50} & 0.393 \\
MVDR-LMS & & 0.430 \\
MVDR & & 0.811 \\
LMS & \multirow{2}{*}{10} & 0.391 \\
MVDR-LMS & & 0.410 \\
MVDR & & 0.810 \\
LMS & \multirow{2}{*}{1} & 0.385 \\
MVDR-LMS & & 0.388 \\
\hline
\end{tabular}

Increasing the number of snapshots will increase the time required to generate beam form due to increase in the time required for training in LMS and MVDR-LMS. While MVDR time to generate beam toward each user is not affected with increasing the number of snapshots, since it doesn't require any training. Although MVDR-LMS require computing beam weights using same equation used in MVDR, then goes through several iteration to keep track of incoming signal. It still can perform good timing compared to traditional LMS. As shown in Table 1 the time required to compute weight in MVDR equal 0.811 seconds. This means MVDR-LMS takes 0.003 seconds more than traditional LMS to generate beams with power higher than that of LMS. 


\section{CONCLUSION}

With the increasing number of mobile systems and fast change in their direction, LTE-5G requires tracking beamformers with maximized power toward each user. Ignoring interference sources that consumes power is also another issue. The available adaptive beamforming algorithms used to produce weights of forming and steering the beam to each individual user has some bugs that make it incompatible with 5G. The MVDR-LMS BF presents beams for multi-users with maximum power thin beams towards targets. These peaks have power less than that of MVDR by $10.2 \%$ and with side lobes power below 100dB. MVDR-LMS can presents beam even when the number of snapshots is reduced to 10 snapshots.

Increasing the number of snapshots will increase the time required to generate beamform due to increase in the time required for training in LMS and MVDR-LMS. While MVDR time to generate beam toward each user is not affected with increasing the number of snapshots, since it doesn't require any training. Although MVDR-LMS require computing beam weights using same equation used in MVDR, then goes through several iteration to keep track of incoming signal. It still can perform good with 3 ms less than traditional LMS.

\section{REFERENCES}

[1] Chen, X., Lu, J., Fan, P.: 'Massive MIMO beam-forming for high speed train communication: directivity vs beamwidth', 2017, arXiv preprint arXiv: 1702.02121.

[2] G. Charis* and N. Showme," Beamforming in Wireless Communication Standards: A Survey", Indian Journal of Science and Technology, Vol 10, issue 5, 2017.

[3] Anila Jose, "Simulation of an Adaptive Digital Beamformer using Matlab", International Journal of Advanced Research in Basic Engineering Sciences and Technology (IJARBEST) Vol. 2, Issue 12, December 2016.

[4] Piya Pal and P. P. Vaidyanathan," Frequency Invariant MVDR Beamforming without filters and Implementation using MIMO radar", 2009 IEEE International Conference on Acoustics, Speech and Signal Processing, April 2009

[5] Ehab ALI, et.al, "Beamforming techniques for massive MIMO systems in 5G: overview, classification, and trends for future research", Frontiers of Information Technology \& Electronic Engineering, Volume 18, Issue 6, pp 753772, June 2017, ISSN 2095-9184 (print); ISSN 2095-9230 (online).

[6] Subrina Biswal, and Deepak Kumar Barik,"Analysis of Adaptive Beam forming Algorithms using LMS and RLS Scheme", IOSR Journal of Electronics and Communication Engineering (IOSR-JECE) e-ISSN: 2278-2834,pISSN: 2278-8735. PP 26-30, 2016.

[7] Debendra Kumar Panda, "DRLMS Adaptive Beamforming Algorithm for Smart Antenna System", International Journal of Applied Engineering Research ISSN 0973-4562 Volume 13, Number 8, pp. 5585-5588, 2018.

[8] Balasem. S.S, et.al, "Beamforming Algorithms Technique by Using MVDR and LCMV", World Applied Programming, Vol. (2), Issue (5), May 2012. 315-324, Special section for proceeding of International E-Conference on Information Technology and Applications (IECITA) 2012.

[9] Sidi Bahri and Fethi Bendimerad," Performance of Adaptive Beamforming Algorithm for LMS-MCCDMA MIMO Smart Antennas", The International Arab Journal of Information Technology, Vol. 6, No. 3, July 2009.

[10] Sohail Payami, "Hybrid Beamforming for Massive MIMO -A Survey", Ph.D. thesis, Institute for Communication Systems Faculty of Engineering and Physical Sciences University of Surrey, May 2017.

[11] Shajahan Kutty, and Debarati Sen, "Beamforming for Millimeter Wave Communications: An Inclusive Survey", IEEE Communications Surveys \& Tutorials, DOI: 10.1109/COMST.2015.2504600, Volume: 18, Issue: 2, pp. $949-973,2016$.

[12] F. Rusek, et aI., "Scaling up MIMO: Opportunities and Challenges with Very Large Arrays," IEEE Signal Processing Mag., vol. 30, no. I, pp. 40-46, Jan. 2013

[13] S. N. Shahab, A. R. Zainun, H. Ahmed Ali, M. Hojabri, and N. H. Noordin, "MVDR algorithm based linear antenna array performance assessment for adaptive beamforming application,” J. Eng. Sci. Technol., vol. 12, no. 5, pp. 1366-1385, 2017.

[14] Song Noh, et.al, "Training sequence design for feedback assisted hybrid beamforming in massive MIMO systems", IEEE Transactions On Communications, VOL. 64, NO. 1, pp. 187-200, JANUARY 2016.

[15] Suhail Najm Shahab, et.al, "Mvdr Algorithm Based Linear Antenna Array Performance Assessment For Adaptive Beamforming Application", Journal of Engineering Science and Technology Vol. 12, No. 5, pp. 1366 - 1385, 2017.

[16] E. A. P. Habets, et.al, "New Insights Into the MVDR Beamformer in Room Acoustics", IEEE Transactions On Audio, Speech, And Language Processing, VOL. 18, NO. 1, pp. 158-170, JANUARY 2010.

[17] Prasanth .CR,"Beam forming and Adaptive beam forming Techniques and its implementation on ADSP TS 201 Processor ",IOSR Journal of VLSI and Signal Processing (IOSR-JVSP) Volume 3, Issue 5 (Nov. - Dec. 2013), PP 07-17

[18] Kalamani M, et.al, "Modified Least Mean Square adaptive Noise Reduction algorithm for Tamil Speech Signal under Noisy Environments", International Journal of Pure and Applied Mathematics, Volume 119 No. 16, pp. 4461-4466, 2018.

[19] Yasmine Mazin Tabra,and Bayan Mahdi Sabar, "Optimal Algorithm For Estimation Of Mobile Users Direction In 5G LTE”, i-manager's Journal on Mobile Applications \& Technologies, Vol. 3, No. 2, May - July 2016. 
[20] Joseph Lardies, et. al, "Power estimation of acoustical sources by an array of microphones", Soci_et_e Fran_caise d'Acoustique. Acoustics 2012, Apr 2012, Nantes, France. 2012.

[21] A. P. Rao, N. V. S. N. Sarma, "Adaptive Beamforming Algorithms for Smart Antenna Systems", WSEAS Transaction on communications, pp.-44-50, vol. 13, 2014.

[22] Revati Joshi, and Ashwinikumar Dhande, "Adaptive Beamforming Using Lms Algorithm”, International Journal of Research in Engineering and Technology, Volume: 03, Issue: 05, May-2014.

[23] Shubhra Dixit, and Deepak Nagaria, "LMS Adaptive Filters for Noise Cancellation: A Review", International Journal of Electrical and Computer Engineering (IJECE), Vol. 7, No. 5, October 2017, pp. 2520-2529.

[24] M. Sowjanya, A.K. Sahoo, Sananda Kumar, " Distributed Incremental Leaky LMS", International conference on Communications and Signal Processing (ICCSP), Melmaruvathur, pp.-1753-1757, Nov. 2015.

[25] Bayan Mahdi Sabar, and Yasmine M. Tabra, "Initial Phase Effect on DOA Estimation in MMIMO Using Separated Steering Matrix”, TELKOMNIKA, Vol.16, No.3, pp. 946 954, June 2018.

\section{BIOGRAPHIES OF AUTHORS}
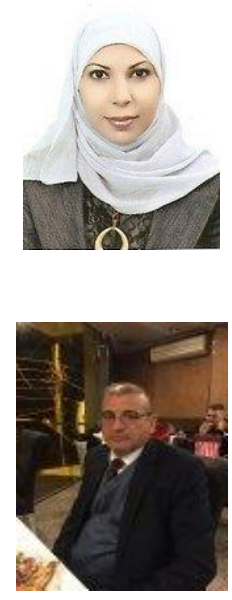

Yasmine Mazin Tabra received her B.Sc. Degree and M.Sc. Degree in Information Engineering from Al-Nahrain University, Baghdad, Iraq in 2005 and 2009. Since 2009, she has been working as a Lecturer in Information and Communication Department/Information Engineering College/ Al-Nahrain University. Currently, she is studying Ph.D. in Information and Communication Engineering. Her fields of interest are Mobile technology, Massive MIMO, Applications of Signal Processing in Direction Finding, beamforming, Image Processing, and Multimedia Communication.

Dr. Bayan Mahdi Sabbar received his B.Sc. in Electrical Engineering, in 1980, M.Sc. in Digital Communications Systems, in 1983, and Ph.D in (Electrical Engineering)/ high resolution array Signal Processing, in 1987. Currently, he is a head of Control Engineerin Department, AlNahrain University, Baghdad, Iraq. His Fields of Interest are Adaptive systems, High resolution algorithms and their applications, Applications of Signal Processing in Direction Finding and Image Processing, and Identifications Systems based on Signal Processing. 